\title{
Modelling the excitation force of a standard tapping machine on lightweight floor structures
}

\author{
Andreas Rabold ${ }^{3 *}$, Martin Buchschmid ${ }^{2}$, Alexander Düster ${ }^{4}$, \\ Gerhard Müller' ${ }^{2}$, Ernst Rank ${ }^{1}$ \\ ${ }^{1}$ Lehrstuhl für Computation in Engineering \\ ${ }^{2}$ Lehrstuhl für Baumechanik, Department of Civil Engineering and Geodesy, \\ Technische Universität München, Arcisstr. 21, 80290 München, Germany \\ ${ }_{3}^{3}$ ift Centre for Acoustics, Theodor-Gietl-Str. 7-9, 83026 Rosenheim, Germany \\ ${ }^{4}$ Numerische Strukturanalyse mit Anwendungen in der Schiffstechnik (M-10), Technische \\ Universität Hamburg-Harburg, Schwarzenbergstr. 95c, 21073 Hamburg, Germany
}

\begin{abstract}
Up to now the research and development in the field of building acoustics is based mainly on measurements. In consequence the development and optimization of a new building component is a very tedious and expensive task. A considerable reduction of these costs could be achieved, if the optimization relying on measurements would be replaced - at least to some extent - by a computational prediction model. For these models it is necessary to represent not only the component and the adjacent rooms but also the excitation in a suitable way. This paper gives an overview of models for the excitation generated by a standard tapping machine taking into account the interaction between the impacting steel cylinders of the tapping machine and the vibrating surface of the floor.
\end{abstract}

Key words: Standard tapping machine, excitation force, lightweight floor, interaction, improved excitation model

\section{INTRODUCTION}

In order to meet the sound insulation requirements of modern living, the optimization of lightweight floor structures is necessary. Until now this process has mainly been based on measurements, using a standard tapping machine which excites the floor according to ISO 140-6 [1]. To avoid tedious setups of measurements it is inevitable for optimization processes to develop a detailed description of the excitation with the help of numerical simulations. Several approaches for modeling the excitation of a standard 
tapping machine have already been published. These range from very simple descriptions of the excitation force by an impulse to current models which include the complete admittance of the floor and the excitation source. Nevertheless all these models neglect the relative velocity between the exciting hammer and the vibrating structure, which leads to significant errors in the case of lightweight floor structures. This paper will first give an overview of these models, and then introduce a modified model, developed in a recent project [2], which considers the time dependency of the excitation process until the floor-vibrations reach a steady state. The findings in the time domain were used for a simplified model in the frequency domain which considers the interaction between the impacting hammer and the vibrating floor. Therefore, the improved model shows a significantly higher degree of accuracy than previous publications. This approach to excitation can be used in a computational optimization model, in which the structural components interact with adjacent rooms in order to consider the effect of room resonances due to the coupling with the acoustic volume.

This paper is organized as follows. Section 2 gives an overview of the most important excitation models building on each other. Section 3 describes the improved model, first in the time domain then in the frequency domain. Section 4 compares the improved model and the measurement results. The discussion of the model is placed in section 5 . Section 6 concludes by summarizing the main features of the proposed model.

\section{THEORY}

\subsection{Model based on Cremer}

The tapping machine consists of 5 steel cylinders (hammers) falling down onto the floor from a height $h=0.04 \mathrm{~m}$ with an (overall) frequency of $f_{s}=1 / T=10 \mathrm{~Hz}$. The hammer impact speed results from the free fall as follows: $\hat{v}_{0}=\sqrt{2 g h}=0.886 \mathrm{~ms}^{-1}$. The impulse resulting from the hammer impact excites the floor vibrations generating the impact sound transmission through the floor. Figure 1 shows a standard tapping machine as well as the time history and the spectrum of the excitation force according to Cremer [3].

(a)

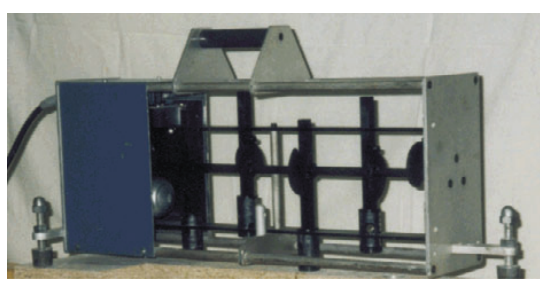

(b)

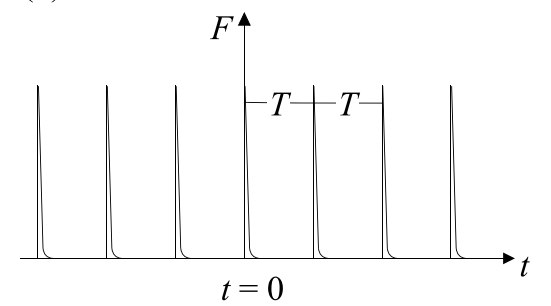

(c)

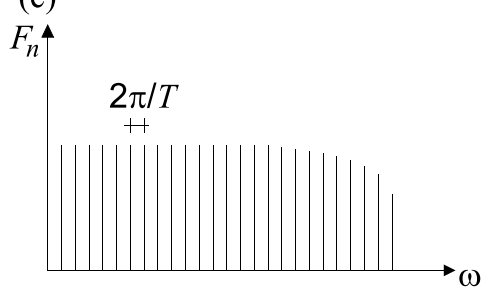

Figure 1. Standard tapping machine (a) with time history (b) and spectrum (c) of the excitation force according to [3]

A Fourier series (equation (1)) is used to develop the time response of the excitation force $F(t)$. The timing $t=0$ is chosen in such a way, that the maximum of the excitation force occurs precisely at that moment. Assuming a symmetric time history it is possible to reduce the Fourier series to its cosine components. ${ }^{1}$

\footnotetext{
${ }^{1}$ The factor 2 in eqn(1), which results from the reduction of the double-sided sum $(-\infty<n<\infty)$ to a one-sided sum $(0<n<\infty)$, in [3] was included in the calculation of the Fourier coefficients (see eqn(2)).
} 


$$
F(t)=\operatorname{Re}\left\{\sum_{-\infty}^{\infty} F_{n} \mathrm{e}^{\mathrm{j} \omega_{n} t}\right\}=\sum_{-\infty}^{\infty} F_{n} \cos \left(\omega_{n} t\right)=2 \sum_{n=1}^{\infty} F_{n} \cos \left(\omega_{n} t\right)+F_{0}
$$

The circular frequency $\omega_{n}$ is expressed as $\omega_{n}=n 2 \pi / T$. The Fourier coefficients $F_{n}$ are determined as in equation (2):

$$
F_{n}=\frac{1}{T} \int_{0}^{T} F(t) \cos \left(\omega_{n} t\right) \mathrm{d} t
$$

If the contact period of the hammer with the floor is very short compared to the time period between two impacts, then $F(t)$ is already sufficiently decayed before $\cos \left(\omega_{n} t\right)$ is significantly different from +1 . This means that the Fourier coefficients can be expressed as the momentum $I$ of the steel cylinder.

$$
F_{n} \approx \frac{1}{T} \int_{0}^{T} F(t) \mathrm{d} t=\frac{I}{T}
$$

It follows that the amplitude of the excitation force $F_{n}$ is constant over the frequency. According to [3] this approximation can only be applied up to frequency $f \approx 1 / T_{c}$, whereby $T_{c}$ represents the contact time between the hammer's and the floor surface. The momentum of the steel cylinder results from its mass $M_{0}$ and the speed at the point of impact $\hat{v}_{0}$. The lower limit $\left(I=M_{0} \hat{v}_{0}\right)$ applies for ideal plastic impacts, the upper limit $\left(I=2 M_{0} \hat{v}_{0}\right)$ for ideal elastic impacts.

The approximation according to [3] is well suited for floors with low admittances (mobilities) and hard surfaces, as those are likely to show less reaction effect to the impact of the hammer.

A comprehensive description of the impact process over time, without the restrictions mentioned above, can also be found in [3] for the impact of a ball on a plate. It is used as the basis for the improved model in the time domain described hereinafter. In this context, the calculation of the Fourier coefficients of the force spectrum is not limited to a certain impact process but can be carried out for nearly any excitation as long as its time response is known. Table 1 shows examples of the time and frequency paths of the excitation force for various excitations.

\subsection{Model based on Vér}

A solution for elastic floor surfaces with the contact stiffness $K_{c}$ was proposed by [4] who deduced the force versus time path from the velocity of an impacting hammer (4) which he assumed to be known.

The hammer impacts the floor with a impact speed of $\hat{v}_{0}$, remains in contact with the floor surface during the impact $F(t)$ for half a vibration period and then leaves the floor with the same speed (in the opposite direction). The contact period $T_{c}$ is calculated as in equation (5) from the contact stiffness $K_{c}$ of the floor surface and the mass of the hammer $M_{0}$. After the contact period $\left(t>T_{c} / 2\right)$ the value for $F(t)=0$. 


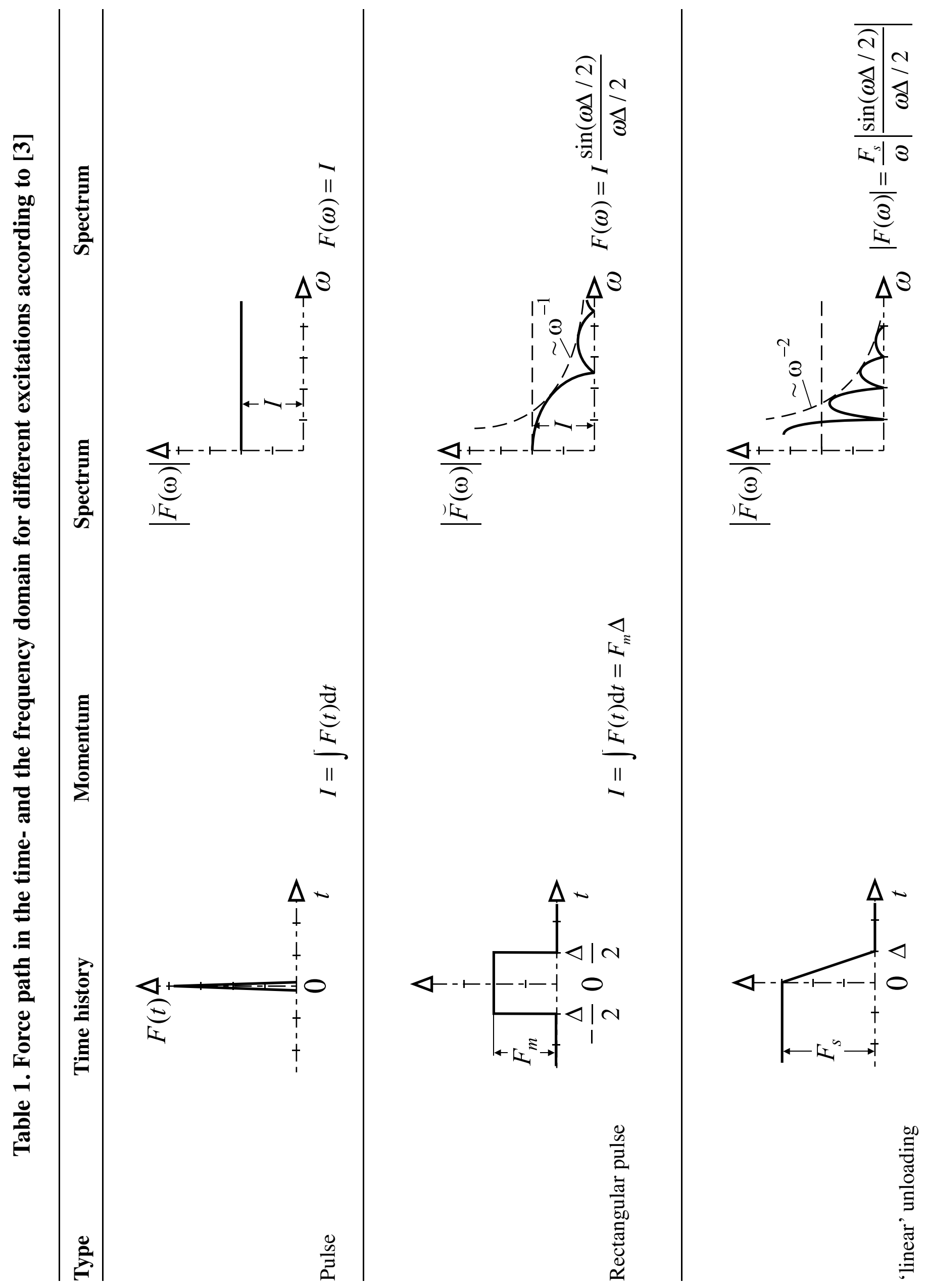



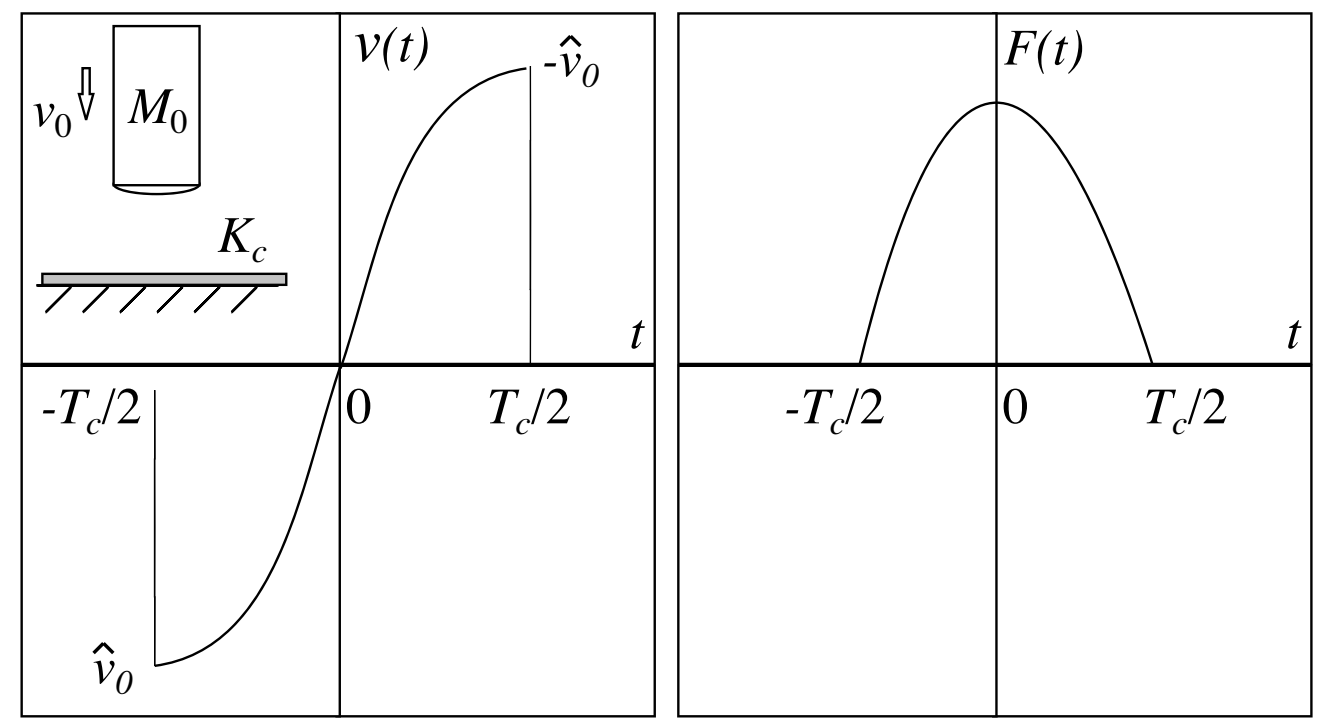

Figure 2. Derivation of the excitation force $F(t)$ from the velocity path $v(t)$

$$
\begin{gathered}
F(t)=M_{0} \hat{v}_{0} \omega_{c} \cos \left(\omega_{c} t\right) \\
\omega_{c}=\sqrt{\frac{K_{c}}{M_{0}}} ; T_{c}=\frac{1}{2} \frac{2 \pi}{\omega_{c}}
\end{gathered}
$$

Inserting equation (4) in equation (2) and integrating over the contact period yields:

$$
F_{n}=\frac{1}{T} \int_{-T_{c} / 2}^{T_{c} / 2} M_{0} \hat{v}_{0} \omega_{c} \cos \left(\omega_{c} t\right) \cos \left(\omega_{n} t\right) \mathrm{d} t
$$

Just like the approximation according to Cremer the practical application of this model is restricted to floors with low admittances as these are not reflected in the model. For these floor admittances to be taken into account, the following models work with appropriate impedances at the interface.

\subsection{Model based on Scholl and Maysenhölder}

Scholl and Maysenhölder [5] applied the above-mentioned models to light-weight floors with a higher admittance. The mass of the floor $M_{1}$ is considered in the calculation of the circular eigenfrequency as in equation (7). In addition to the contact stiffness $K_{c}$ of the floor surface, the stiffness of the excitation $K_{0}$ is included in order to be able to model also other excitations (rubber ball, human foot), as well as those from the tapping machine.

$$
\omega_{c}=\sqrt{\frac{K}{M}} ; \quad K=\frac{1}{\frac{1}{K_{c}}+\frac{1}{K_{0}}} ; \quad M=\frac{1}{\frac{1}{M_{0}}+\frac{1}{M_{1}}}
$$




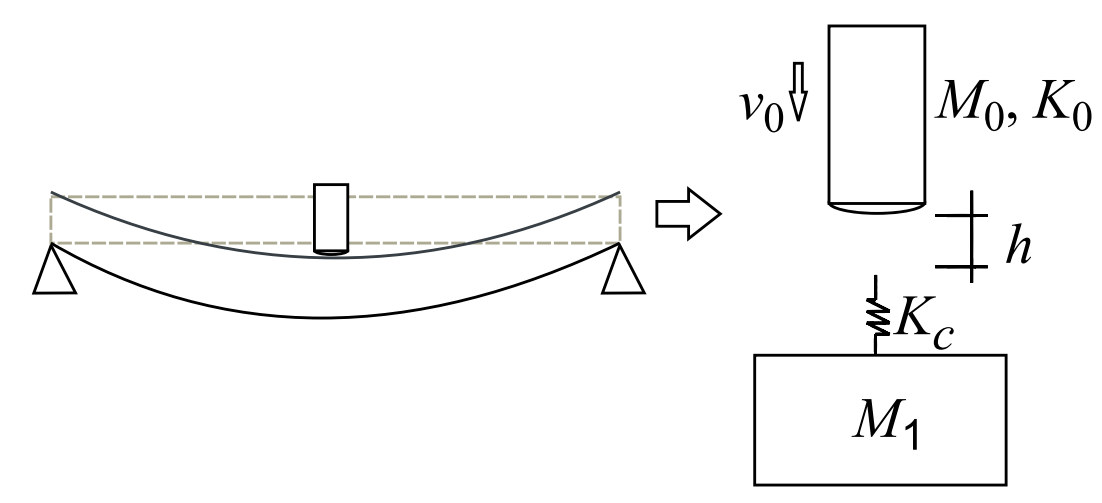

Figure 3. Model for the excitation of light-weight floors according to [5]

According to [3] the contact stiffness $K_{c}$ of the floor surface is determined using the shear modulus $G$ and the Poisson ratio $\mu$ of the floor surface, as well as the radius $r$ of the hammer (diameter of the cylinder):

$$
K_{c} \approx a \frac{G r}{1-\mu} ; a=\pi, \ldots, 4
$$

A more detailed discussion of the contact stiffness and the contact area can be found in $[6,7]$.

The model focuses on the description of the contact stiffness of the floor surface and different excitations, which made it possible to compare the excitation of a floor by a standard tapping machine to that by a walking person. The floor itself is included only with its overall mass and is thus inadequately described as the participation of the floor mass in the different eigenmodes varies. The stiffness and damping of the floor are not taken into account.
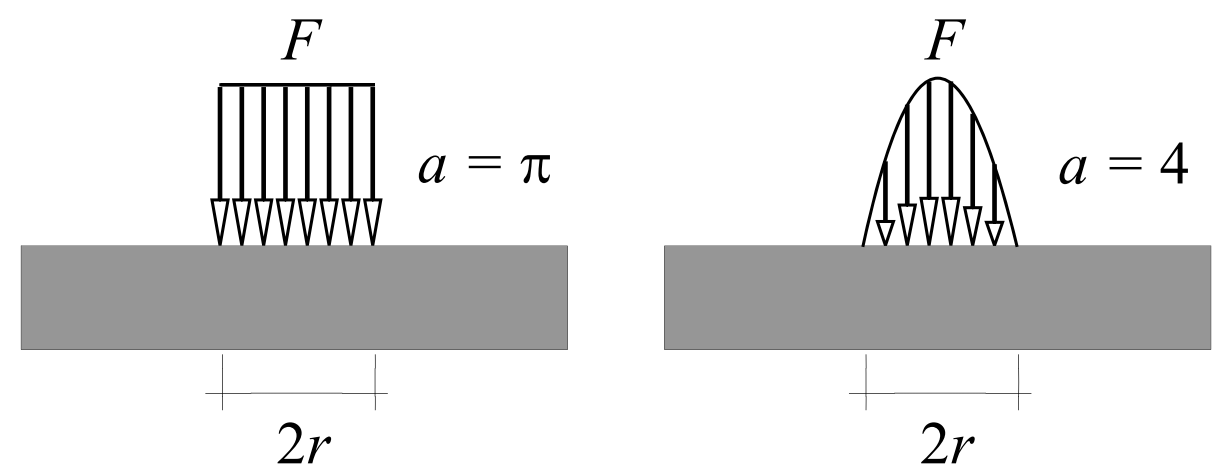

Figure 4. Form factor for calculating the contact stiffness in relation to the force distribution during the excitation

\subsection{Model based on Brunskog and Hammer}

Brunskog and Hammer [8] have developed a model for the spectrum of the excitation which takes the admittance of the floor fully into account. The excitation force is derived from the equilibrium of forces during the contact of the hammer with the floor (Figure 5 (a) - (c)). In order to obtain a time-invariant description, the mass of the hammer $\left(M_{0}\right)$ in the transition from Figure 5(b) to Figure 5(c) is considered as firmly 
fixed to the floor, and the excitation is carried out with the as yet unknown impact force $F_{0}$. In this case, the admittance $Y$ of the system is composed of a local and a global part.

(a)
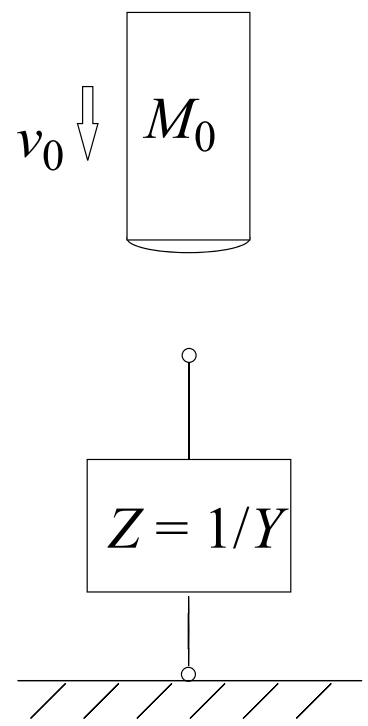

(b)

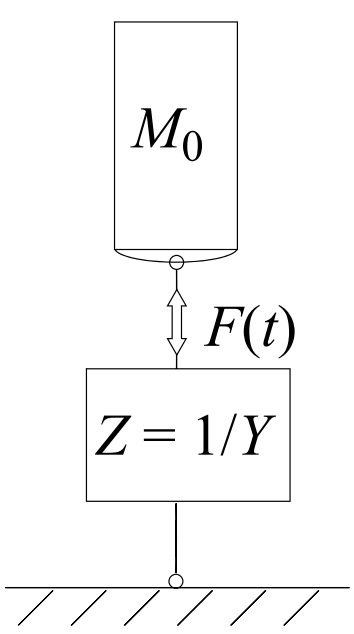

(c)

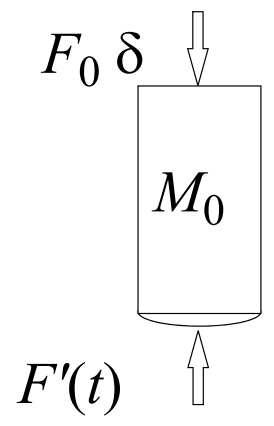

Figure 5. Model for the excitation of a floor by the impact from a tapping machine according to [8]

With the Fourier coefficients $F_{n}^{\prime}(\omega, n)$ of the time-invariant reaction force $F^{\prime}(t)$ of the floor and the inertia force $M_{o j} \omega_{n} v$ of the hammer, the following equation results in the frequency range:

$$
F^{\prime}(t)=\sum_{n=-\infty}^{\infty} F_{n}^{\prime} e^{j \omega_{n} t}, \quad F_{0, n}-F_{n}^{\prime}=\mathrm{j} \omega_{n} M_{0} v
$$

Considering $v=F_{n}^{\prime} Y$ in equation, $F_{n}^{\prime}$ results in:

$$
F_{n}^{\prime}=\frac{F_{0, n}}{1+\mathrm{j} \omega_{n} M_{0} Y}
$$

The value for $F_{0}$ results from the postulate that, directly after the Dirac impulse, $M_{0}$ moves with the initial velocity $v(0)=\hat{v}_{0}$, i.e. with the hammer's fall velocity directly before the impact. As only half of the (infinitesimally short) impulse is in the time interval under consideration $(t>0)$, in [8] it is assumed for the calculation that the actual initial velocity is $v(0)=\hat{v}_{0} / 2$ :

$$
v(t=0)=\frac{1}{2 \pi} \int_{-\infty}^{\infty} v(\omega) \mathrm{d} \omega=\hat{v}_{0} / 2
$$

For the calculation of the actual reaction force $F_{n}, F_{n}^{\prime}$ is inverse transformed into the time domain and its force/time path is cut off at the first zero-crossing of the force function - which coincides with the hammer lifting off from the floor. Now the part up to the first zero-crossing is inverse transformed into the frequency range, resulting in $F_{n}$. 
It follows that this model can be applied to all types of floors and floor finishes since not only the global admittances of the floors are taken into account but also the local admittances of the floor surfaces. An overview of possible applications of the excitation models described above is shown in Table 2.

\section{Table 2. Overview of possible applications of the excitation models described above}

\begin{tabular}{lcccc}
\hline & \multicolumn{3}{c}{ Model based on: } \\
\hline & $\begin{array}{c}\text { Cremer } \\
{[3]}\end{array}$ & $\begin{array}{c}\text { Vér } \\
{[4]}\end{array}$ & $\begin{array}{c}\text { Scholl } \\
\text { et al. [5] }\end{array}$ & $\begin{array}{c}\text { Brunskog } \\
\text { et al. [8] }\end{array}$ \\
\hline $\begin{array}{l}\text { floor with low admittance }\left(Y_{G} \rightarrow 0\right) \\
\text { and hard surface }\left(T_{c} \rightarrow 0\right)\end{array}$ & & & & \\
\hline $\begin{array}{l}\text { floor with low admittance }\left(Y_{G} \rightarrow 0\right) \\
\text { and elastic component surface }\left(T_{c} \rightarrow 0\right)\end{array}$ & & & & 0 \\
\hline $\begin{array}{l}\text { excitation with different contact } \\
\text { stiffness } K_{c} \text { (hammer - foot) }\end{array}$ & & & \\
\hline floor with higher admittance $\left(Y_{G}>>0\right)$ & & & 0 \\
\hline
\end{tabular}

However, the excitation spectra of the tapping machine on lightweight floors calculated in accordance with Brunskog and Hammer do not show the expected correlation to measured values. The computed results are very close to the limit values for the ideal plastic impact (see [8]). This does not agree with measured values for lightweight floors [10] which show significantly higher values in the excitation spectrum. A possible reason for these discrepancies is the approximation of the initial velocity $v(0)=\hat{v}_{0} / 2$ and the assumption that the component surface is at rest before the impact. The impact sound transmission is measured when the floor is in its steady state. Therefore, a relative velocity between the impacting hammer and the vibrating floor (due to previous hammer impacts) must be expected. This effect shall be studied in more detail in the following chapter.

\section{MODEL IMPROVEMENT}

\subsection{Modelling the excitation force in the time domain}

In order to get a better understanding of the interaction between the floor and the impacting hammer, we first take a look at the impact in the time domain. Based on the results in the time domain, the calculation in the frequency domain will be extended to include the phenomenon of the relative velocity between the floor and the hammer.

For the time-related modelling of the impact it is possible to use a model by Cremer and Heckl [3] which describes the impact of a ball on a plate with an additional flexible covering. Figure 6 shows the model applied to an excitation of a light-weight floor by a tapping machine (with additional damping at the point of contact taken into account).

The response of the floor is described by a global part (admittance $Y_{G}$ of the floor) and the deformation part at the point of impact (local admittance $Y_{L}$ ). The local admittance is the result of the mass of the hammer $M_{0}$, the damping $C_{c}$ and the stiffness 

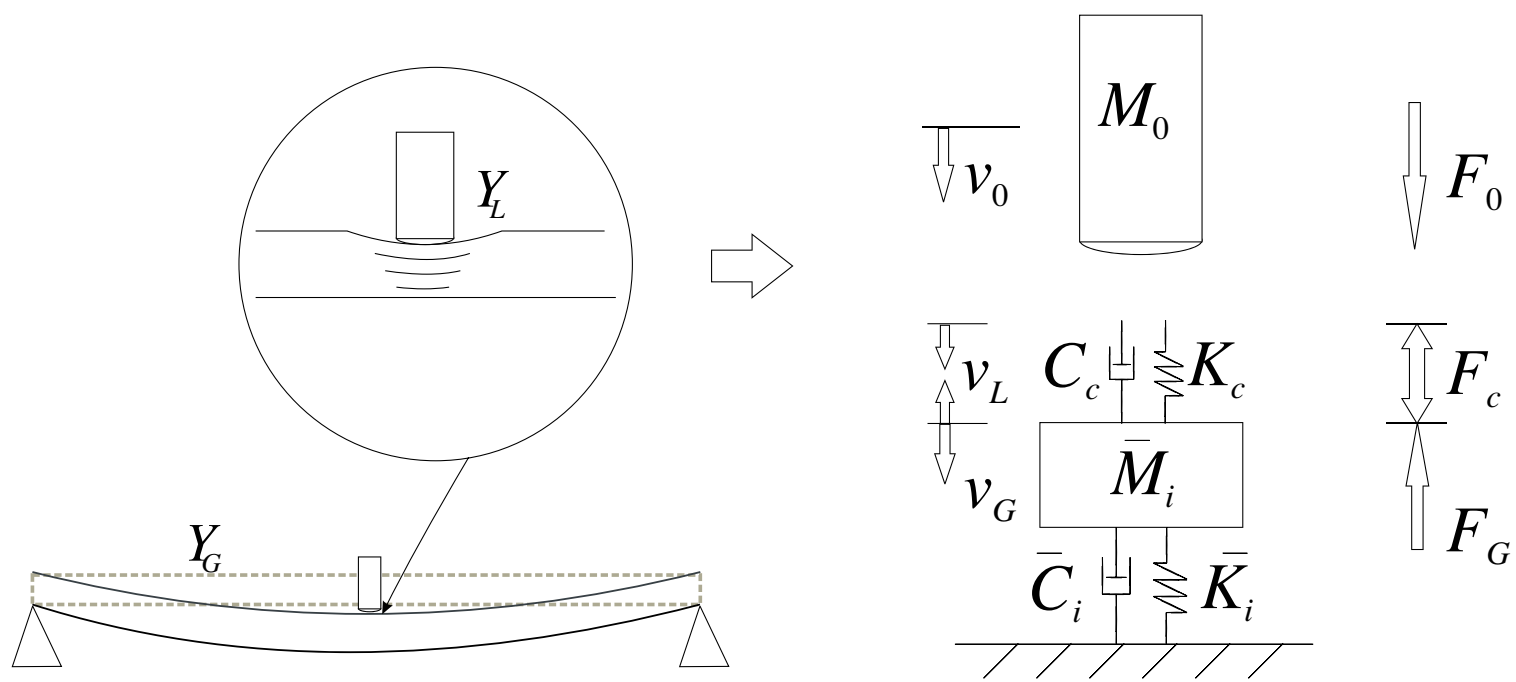

Figure 6. Improved model for the excitation of the $i$ different eigenmodes of a floor by the hammer impact from a tapping machine $(i=1,2,3, \ldots, n)$

$K_{c}$ at the point of contact. The global admittance results from the sum of modal mass $\bar{M}_{i}$, damping $\bar{C}_{i}$ and stiffness $\bar{K}_{i}$ values.

Based on the equilibrium of the impacting hammer $F_{0}(t)$ and the counteracting contact force of the floor $F_{c}(t)$, the equation for the time-related velocity of the hammer $v_{0}(t)$ results after applying an integration over time, where $t=0$ marks the time when the hammer is released:

$$
F_{c}(t)=F_{0}(t)=M_{0} g-M_{0} \frac{d v_{0}}{\mathrm{~d} t} \Rightarrow v_{0}(t)=g t-\frac{1}{M_{0}} \int_{0}^{t} F_{c}\left(t_{q}\right) \mathrm{d} t_{q}+C_{i n t}
$$

The integration constant $C_{i n t}$ can be determined from the initial conditions. Since the tapping machine rests on the vibrating floor, the hammer moves - before being released for the drop - initially at the same speed as the floor $\left(v_{G}(t)\right)$. The integration constant can therefore approximately be expressed as $C_{\text {int }}=v_{0, t_{0}}$ with $v_{0, t_{0}}=v_{G}(t=0+(m-1) T)$. For the first impact $(m=1) v_{0, t_{0}}=0$ holds.

In the next step the velocity of the vibrating floor has to be determined. Since the floor surface at the point of contact moves at the same velocity as the hammer, one observes:

$$
v_{0}(t)=v_{L}(t)+v_{G}(t)
$$

The velocity of the floor surface consists of the local deformation component at the point of impact $v_{L}(t)$ and the global component $v_{G}(t)$ of the floor. The local component results from the force of impact $F_{c}(t)$ :

$$
F_{c}(t)=K_{c} \int_{0}^{t} v_{L}\left(t_{q}\right) \mathrm{d} t_{q}+C_{c} v_{L}
$$

The global component of the floor vibration is derived with the help of the modal analysis. In the time domain, the overall vibration can be derived from superposition of the impulse responses for each eigenmode $\varphi_{i}$ of the floor. The real impact at the point 
of excitation $x_{0}, y_{0}$ is composed out of ideal individual impulses using the Duhamel integral [9].

$$
\begin{gathered}
v_{G}(t)=\int_{0}^{t} F_{c}\left(t_{q}\right) \sum_{i=1}^{\infty} \dot{h}_{i}\left(t-t_{q}\right) d t_{q} \\
h_{i}(t)=\frac{1}{M_{i} \omega_{i}} e^{-D_{i} \omega_{i} t} \sin \left(\omega_{i} t\right) ; M_{i}=\frac{\bar{M}_{i}}{\varphi_{i}^{2}\left(x_{0}, y_{0}\right)} ; D_{i}=\frac{C_{i}}{2 M_{i} \omega_{i}} \\
\dot{h}_{i}(t)=\frac{1}{M_{i}} e^{-D_{i} \omega_{i} t}\left[\cos \left(\omega_{i} t\right)-D_{i} \sin \left(\omega_{i} t\right)\right]
\end{gathered}
$$

Thus the problem can be solved numerically for $m$ time steps and $N_{\text {eigen }}$ eigenmodes $\left(\varphi_{i}\right)$ of the floor. Evaluating the velocity of the hammer and the floor surface during the impact, we obtain the following equation for the $\mu$-th period of time $\Delta t$ :

$$
v_{0}(\mu \Delta t)=v_{c}(\mu \Delta t) \quad \text { resp. } v_{0}(\mu \Delta t)=v_{L}(\mu \Delta t)+v_{G}(\mu \Delta t)
$$

After inserting equations (12), (14) and (15) in equation (18) and expressing in discrete time steps, the following equation results:

$$
g \mu \Delta t-\sum_{v=1}^{\mu-1} \frac{F_{c, v}}{M_{0}} \Delta t-\frac{F_{c, \mu}}{M_{0}} \Delta t+v_{0, t_{0}}=\left\lfloor\frac{F_{c, \mu}-K_{c} \sum_{v=1}^{\mu-1} v_{L, v} \Delta t}{C_{c}+K_{c} \Delta t}+\sum_{v=1}^{\mu-1}\left\{F_{c, v} \Delta t \sum_{i=1}^{N_{c i g e n}} \dot{h}_{i}((\mu-v) \Delta t)\right\}\right\rfloor
$$

and after a simple mathematical transposition:

$F_{c, \mu} \Delta t=M_{0}\left[g \mu \Delta t-\sum_{v=1}^{\mu-1} \frac{F_{c, v}}{M_{0}} \Delta t+v_{0, t_{0}}\right]-M_{0}\left[\frac{F_{c, \mu}-K_{c} \sum_{v=1}^{\mu-1} v_{L, v} \Delta t}{C_{c}+K_{c} \Delta t}+\sum_{v=1}^{\mu-1}\left\{F_{c, v} \Delta t \sum_{i=1}^{N_{\text {cigen }}} \dot{h}_{i}((\mu-v) \Delta t)\right\}\right]$

Thus in equation (20) the partial impulse $\left(\Delta I=F_{c, \mu} \Delta t\right)$ is calculated for each time step. The time step $\Delta t$ has to be selected such that the partial impulse can be appropriately modelled by a Dirac impulse. This means that the duration of the impulse must be short enough so that the change of velocity of the floor's vibration resulting from the impulse is negligible during the time step $\left(\Delta v_{G}(\Delta t) \rightarrow 0\right){ }^{2}$

${ }^{2}$ Contrary to this approach, in [3] this is taken account of by assuming a 'half' impulse for the actual time period. 
Using the time-dependent representation of the impact, it is now possible to simplify the previous models. Calculating with equation (20), choosing a very high contact stiffness $\left(K_{c} \rightarrow \infty\right)$ and assuming the admittance of the floor is very small $\left(1 / M_{i} \rightarrow 0\right)$, one obtains the same results as with the original model according to Cremer, which simplifies the complete impact process to a Dirac impulse (Figure 7(a)). If a realistic contact stiffness is taken into account in the calculation, the results fit in with the modelling according to Vér (Figure 7(b)). The change of the vibration velocity of the floor $v_{G}$ during the impact remains negligible due to the low floor admittance. If also the floor admittance (for light-weight floors) is taken into account we arrive at the model by Brunskog and Hammer (Figure 7(c)). If, in addition, the relative velocity between floor and hammer, which is the result of previous impacts, is considered we arrive at Figure 7(d).

(a)

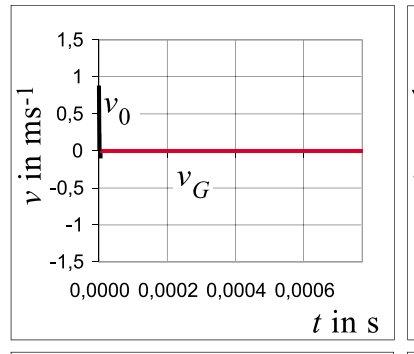

(b)
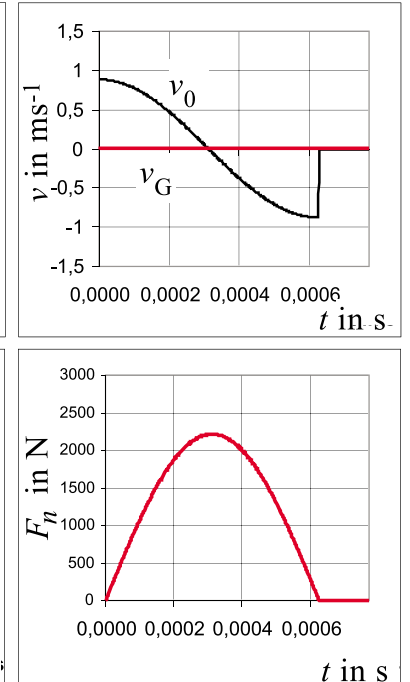

(c)
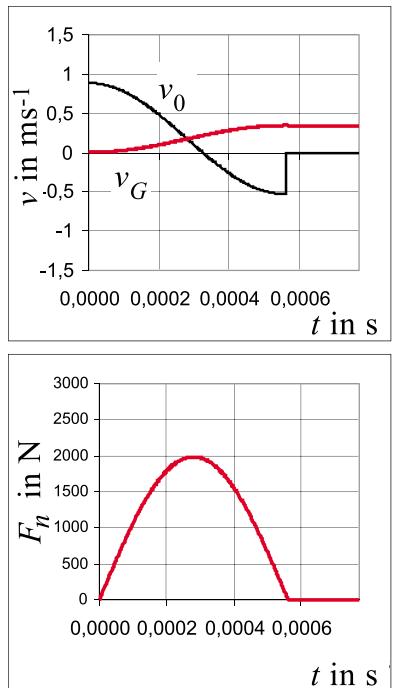

(d)
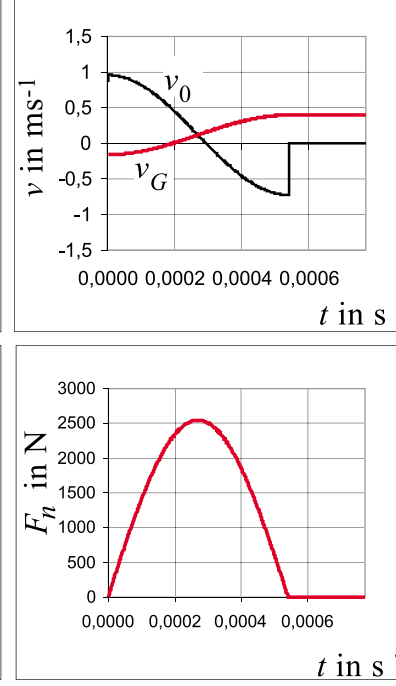

Figure 7. Models of the excitation with different levels of simplification, computed with Equation (20): (a) $K_{c} \rightarrow \infty, 1 / M_{i} \rightarrow 0, v_{G}(\mathrm{t}=0)=0$ (b) $1 / M_{i} \rightarrow 0$, $v_{G}(t=0)=0$ (c) $v_{G}(t=0)=0$ (d) $v_{G}(t=0) \neq 0$

Figure 7(d) shows that the excitation force - depending on the phase shift between the velocities of hammer and floor - can differ significantly from the results of the other models up to now. In the following the influence of the relative velocity is discussed for different types of floors. Table 3 shows an example of the calculation of the vibration velocity at the point of excitation for two eigenmodes and floor types with different admittances.

It follows that the relative velocity between floor and hammer is relevant when the vibration resulting from the previous impact has not yet completely subsided at the time of the next impact. This is primarily the case with light-weight floors (large admittance) and low-frequency vibrations (taking a long time to completely subside).

Now a complete computation in the time domain would be possible for a defined 'measuring interval', followed by an inverse transformation into the frequency domain. However, this procedure involves a considerable numerical effort and requires a high 
low admittance

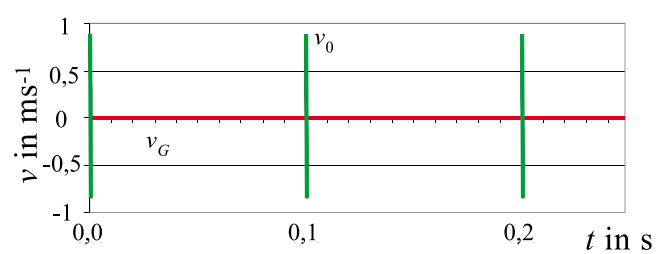

$\omega_{i}=2 \pi 200 \mathrm{~Hz}$

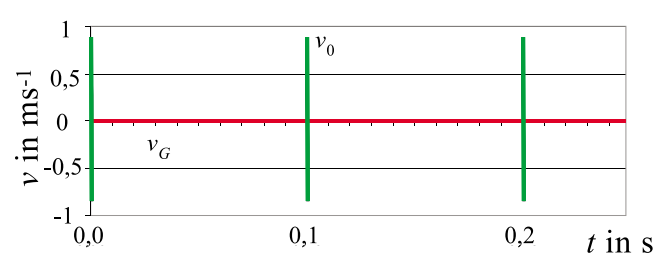

high admittance
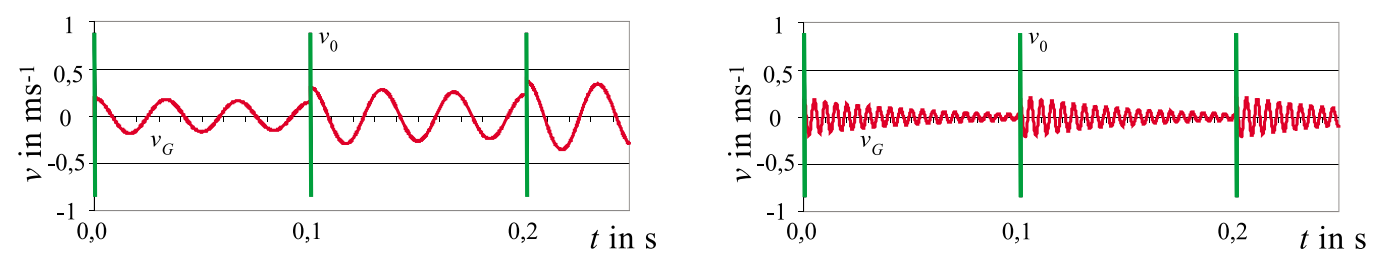

degree of accuracy of the eigenfrequencies in order to represent the relative velocity between floor and hammer correctly. For this reason a simplified computation in the frequency domain is proposed as an alternative.

\subsection{Modelling the excitation force in the frequency domain}

It is possible to describe the interaction between hammer and floor, presented in the time domain, in the frequency domain approximatively in an iterative process. To do that, a time-invariant description in the frequency domain is aimed by the model shown in Figure 6. As in [3] and [8] this is done by introducing an impact force $F_{0, n}$ on the hammer $\left(M_{0}\right)$ which is fixed to the structure, where the index $n$ marks the number of the Fourier coefficient:

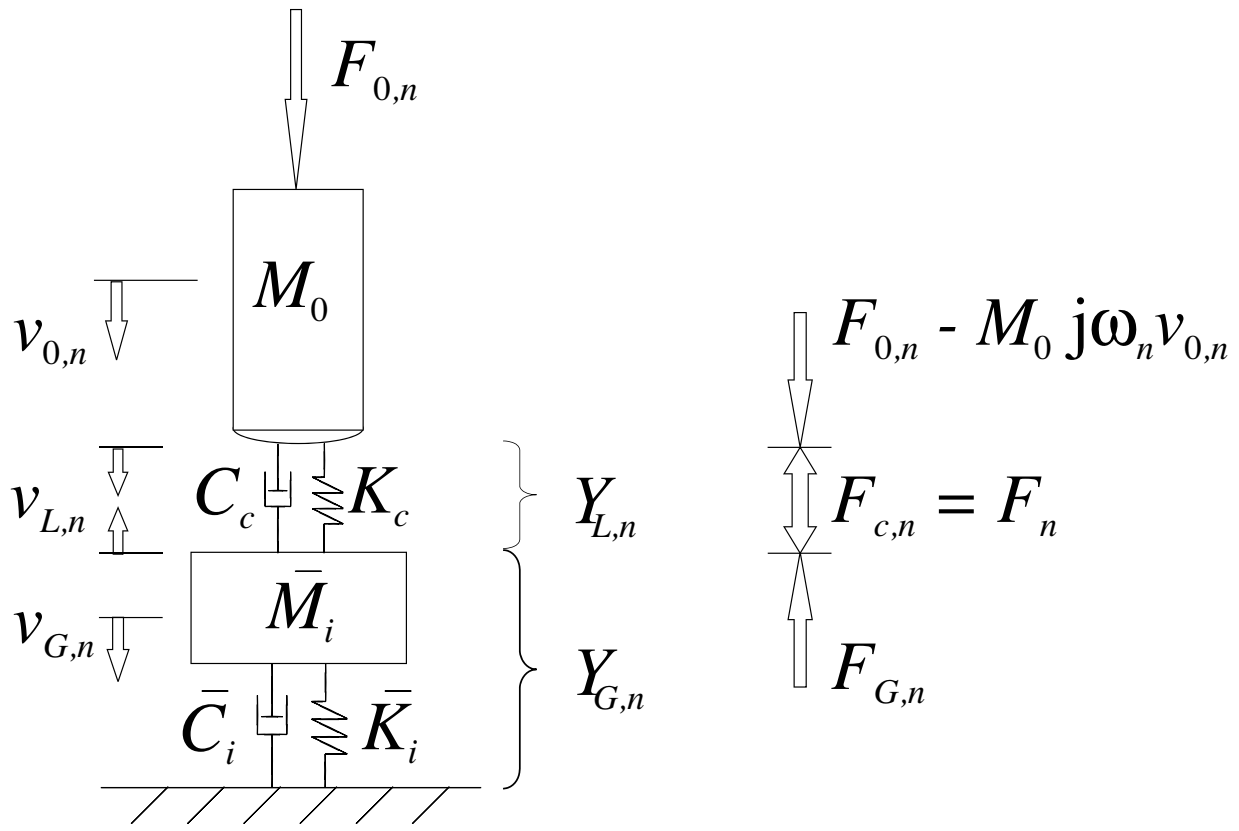

Figure 8. Model for the excitation force in the frequency domain 
From Figure 8 follows:

$$
F_{n}=F_{0, n}-M_{0} \mathrm{j} \omega_{n} v_{0, n} \quad \text { where } v_{0, n}=v_{L, n}+v_{G, n}=F_{n} Y_{L, n}+F_{n} Y_{G, n}
$$

After inserting and rearranging the following equation holds, during the contact:

$$
F_{n}=\underbrace{F_{0, n}-F_{n} Y_{L, n} M_{0} \mathrm{j} \omega_{n}}_{F_{n, L}}-\underbrace{F_{n} Y_{G, n} M_{0} \mathrm{j} \omega_{n}}_{F_{n, G}}
$$

The contact force $F_{n}$ can be split up into a local component $F_{n, L}$ and a global component $F_{n, G} . F_{n}$ is calculated from the force $F_{0, n}$, which is reduced depending on the local admittance $Y_{L, n}$ and the global admittance $Y_{G, n}$. If both, the local and global admittance vanish, then $F_{n}=F_{0, n}$, resulting in $F_{0, n}$ as the spectrum of an ideal elastic impact as shown in the simplified frequency-related model according to Cremer [3]. If only the global admittance vanishes, then $F_{n}=F_{n, L}$ and $F_{n}$ can be calculated using the model according to Vér [4] which takes into account the time variance that has been neglected in equation (22). Here, where both admittances are relevant, the local component $F_{n, L}$ is calculated in the first step using a modified model according to Vér and then the whole force $F_{n}$ is calculated in the steady state with the help of an iterative procedure.

The modification of the model for the local component $F_{n, L}$ requires the inclusion of the relative velocity $v_{R}$ between the impacting hammer and the vibrating floor, described in the previous section, as well as the local damping effect $D_{c}=C_{c} /\left(2 \omega_{c} M_{0}\right)$.

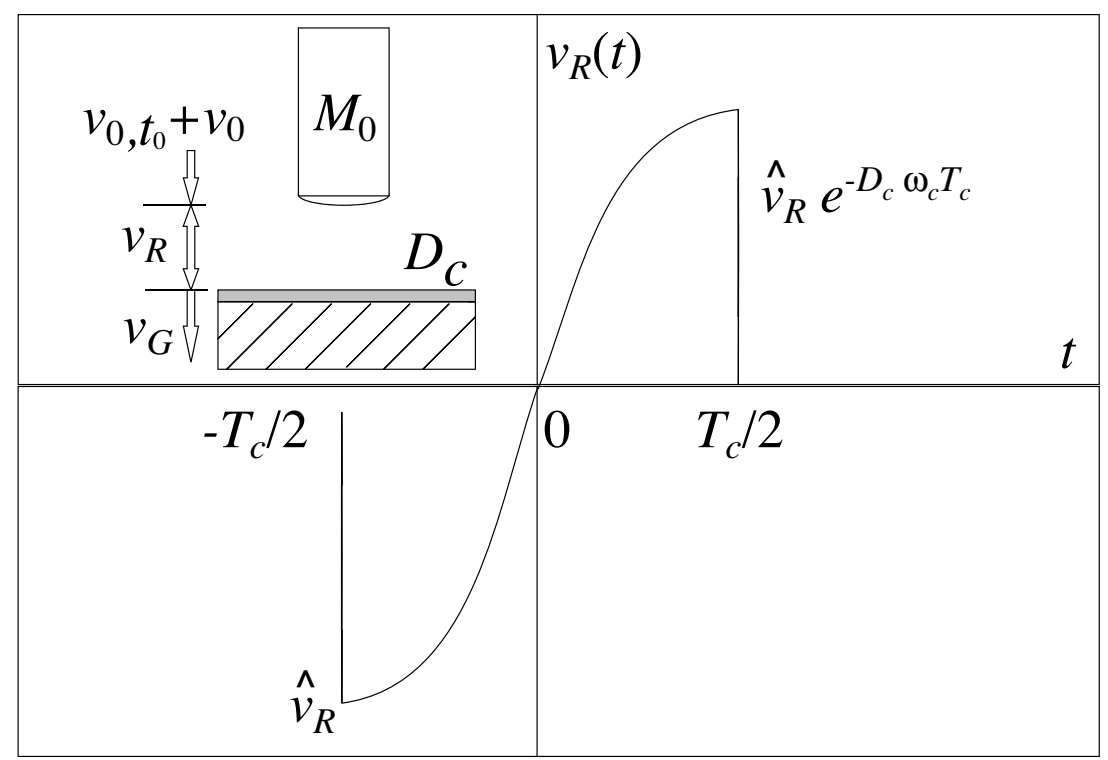

Figure 9. Relative velocity path $v_{R}(t)$

According to Figure 9, the peak value of the relative velocity $\hat{v}_{R}$ of the floor and the impacting hammer is made up of the velocity at the end of the free fall $\hat{v}_{0}$ plus the initial speed of the hammer when released from the mechanism $\hat{v}_{0, t_{0}}$ and also the already existing vibration velocity of the floor $v_{G, m}$ of the $m^{\text {th }}$ impact of the hammer:

$$
\hat{v}_{R, m}=\hat{v}_{0}+v_{0, t_{0}}-v_{G, m}
$$


The relative velocity $v_{R}$ is given as:

$$
v_{R, m}(t)=\hat{v}_{R, m} \mathrm{e}^{-D_{c} \omega_{c} t} \sin \left(\omega_{c} t\right)
$$

From this the local component $F_{n, L, m}$ can be derived for the $m^{\text {th }}$ impact of the hammer:

$$
F_{n, L, m}=\frac{1}{T_{n}} \int_{-T_{n} / 2}^{T_{n} / 2} F_{L}(t) \mathrm{e}^{-\mathrm{j} \omega_{n} t} \mathrm{~d} t=\frac{1}{T_{n}} \int_{-T_{c} / 2}^{T_{c} / 2} M_{0} \dot{v}_{R, m}(t) \mathrm{e}^{-\mathrm{j} \omega_{n} t} \mathrm{~d} t
$$

In equations (24) and (25) the assumption was made, that the contact period $T_{c}=$ $\pi / \omega_{c}$ is not significantly influenced by the global admittance of the floor. $F_{n, L, m}$ is initially calculated for a single impact. For this, $T_{n}$ should be selected such that the vibration of the respective structure has already decayed at the end of the period $T_{n}$. For the structures under consideration in this paper this was $T_{n}=0.5 \mathrm{~s}$, i.e. the interval between the repeated impacts of a (single) hammer. From this can be derived the $n^{\text {th }}$ circular frequency $\omega_{n}=n 2 \pi / T_{n}=n 4 \pi \mathrm{Hz}$.

For the first impact $\hat{v}_{R}=\hat{v}_{0}$ holds. In order to avoid another Fourier transformation in the subsequent steps of computation, it is possible to calculate $F_{n, L, m}$ for the subsequent impacts from the result of the first impact by inserting equation (24) in equation (25):

$$
F_{n, L, m}=\frac{\hat{v}_{R, m}}{\hat{v}_{0}} F_{n, L, 1}
$$

The global component of the excitation force for a structure with $n_{\text {eigen }}$ degrees of freedom is calculated from the global admittance $Y_{G, i}$ and the displacements of the eigenmodes $\varphi_{i}$ of the floor at the point of excitation $\left(x_{0}, y_{0}\right)$ :

$$
F_{n, G}=M_{0} F_{n} \mathrm{j} \omega_{n} \sum_{i=1}^{n_{\text {eigen }}} Y_{G, n, i} \varphi_{i}^{2}\left(x_{0}, y_{0}\right)
$$

For the first impact of the hammer on the floor, which is still at rest, one obtains after inserting equation (27) in equation (28):

$$
F_{n, 1}=\frac{F_{n, L, 1}}{1+M_{0} \mathrm{j} \omega_{n} \sum_{i=1}^{n_{\text {eigen }}} Y_{G, n, i} \varphi_{i}^{2}\left(x_{0}, y_{0}\right)}
$$

From the second to the $m^{\text {th }}$ impact on the structure the relative velocity between the floor, which is now vibrating already, and the impacting hammer have to be taken into account:

$$
F_{n, m}=\frac{\frac{\hat{v}_{R}}{\hat{v}_{0}} F_{n, L, 1}}{1+M_{0} \mathrm{j} \omega_{n} \sum_{i=1}^{n_{\text {eigen }}} Y_{G, n, i} \varphi_{i}^{2}\left(x_{0}, y_{0}\right)}
$$


The relative velocity $\hat{v}_{R}$ is calculated according to equation (23) from the velocity of the hammer at the end of the free fall $\hat{v}_{0}$ plus the initial speed of the hammer when released from the mechanism $v_{0, t_{0}}$ and the already existing velocity of the floor $v_{G, m}$. The velocity $v_{G, m}$ of the floor at the time of impact is obtained by adding the components of the individual eigenmodes. The components of the individual eigenmodes are calculated out of the velocity $v_{G, m-1, \mathrm{i}}$ immediately after the last impact, taking into account the damping $D_{i}$ during the free vibration between two impacts.

$$
v_{G, m}=\sum_{i=1}^{n_{\text {eigen }}} \hat{v}_{G, m-1, i} \mathrm{e}^{-D_{i} \omega_{i} T} \cos \left(\omega_{i} T+\alpha_{G, m-1, i}\right)
$$

In equation (30) $\hat{v}_{G, m-1, i}$ and $\alpha_{G, m-1, i}$ represents the amplitude and the phase of $v_{G, m-1, i}$ respectively. $v_{G, m-1, i}$ is calculated out of the spectral components as follows for the time $T_{c}$ immediately after the last impact (m-1):

$$
v_{G, m-1, i}=2 \sum_{n=1}^{n_{\max }} v_{G, m-1, i, n} \mathrm{e}^{\mathrm{j} \omega_{n} T_{c}}
$$

The Fourier coefficient of the vibration velocity $v_{G, m-1, i, n}$ is calculated from the (still existing) vibration velocity $v_{G, m-2, i, n}$ of previous impacts (from $m>2$ ) and the excitation force $F_{n, m-1}$ of the preceding hammer impact.

$$
v_{G, m-1, i, n}=v_{G, m-2, i, n} \mathrm{e}^{-D_{i} \omega_{i} T} \mathrm{e}^{\mathrm{j} \omega_{i} T}+F_{n, m-1} Y_{G, n, i} \varphi_{i}^{2}\left(x_{0}, y_{0}\right)
$$

The initial velocity $v_{0, t_{0}}$ of the hammer (when it is released from the mechanism) is calculated in the same way for the time of release $\left(T-T_{\text {fall }}\right)$ :

$$
v_{0, t_{0}}=\sum_{i=1}^{n_{\text {cigen }}} \hat{v}_{G, m-1, i} \mathrm{e}^{-D_{i} \omega_{i}\left(T-T_{\text {fall }}\right)} \cos \left(\omega_{i}\left(T-T_{\text {fall }}\right)+\alpha_{G, m-1, i}\right)
$$

The time of the free fall between the release from the mechanism and the impact, for the first impact is: $T_{\text {fall }}=\sqrt{2 s_{0} / g} \approx 0.09 \mathrm{~s}$. For the subsequent impacts $T_{\text {fall }}$ is also assumed to be constant.

The transient process described in equations (25) to (33) is calculated until the structure has reached the steady state. An overview of the iteration scheme used for the calculation is shown in Figure 10.

The amplitude of the vibration velocity $v_{n}\left(x_{l}, y_{l}\right)$ at any point $\left(x_{l}, y_{l}\right)$, which is required for the spectral analysis, can now be calculated directly from the (converged) vibration velocity at the point of excitation $v_{G, i, n}\left(x_{0}, y_{0}\right)$ according to equation (31) and the deflection of the eigenmode $\varphi_{i}$ at point $\left(x_{l}, y_{l}\right)$ :

$$
v_{n}\left(x_{l}, y_{l}\right)=\sum_{i=1}^{n_{\text {eigen }}} \frac{v_{G, i, n}\left(x_{0}, y_{0}\right)}{\varphi_{i}\left(x_{0}, y_{0}\right)} \varphi_{i}\left(x_{l}, y_{l}\right)
$$




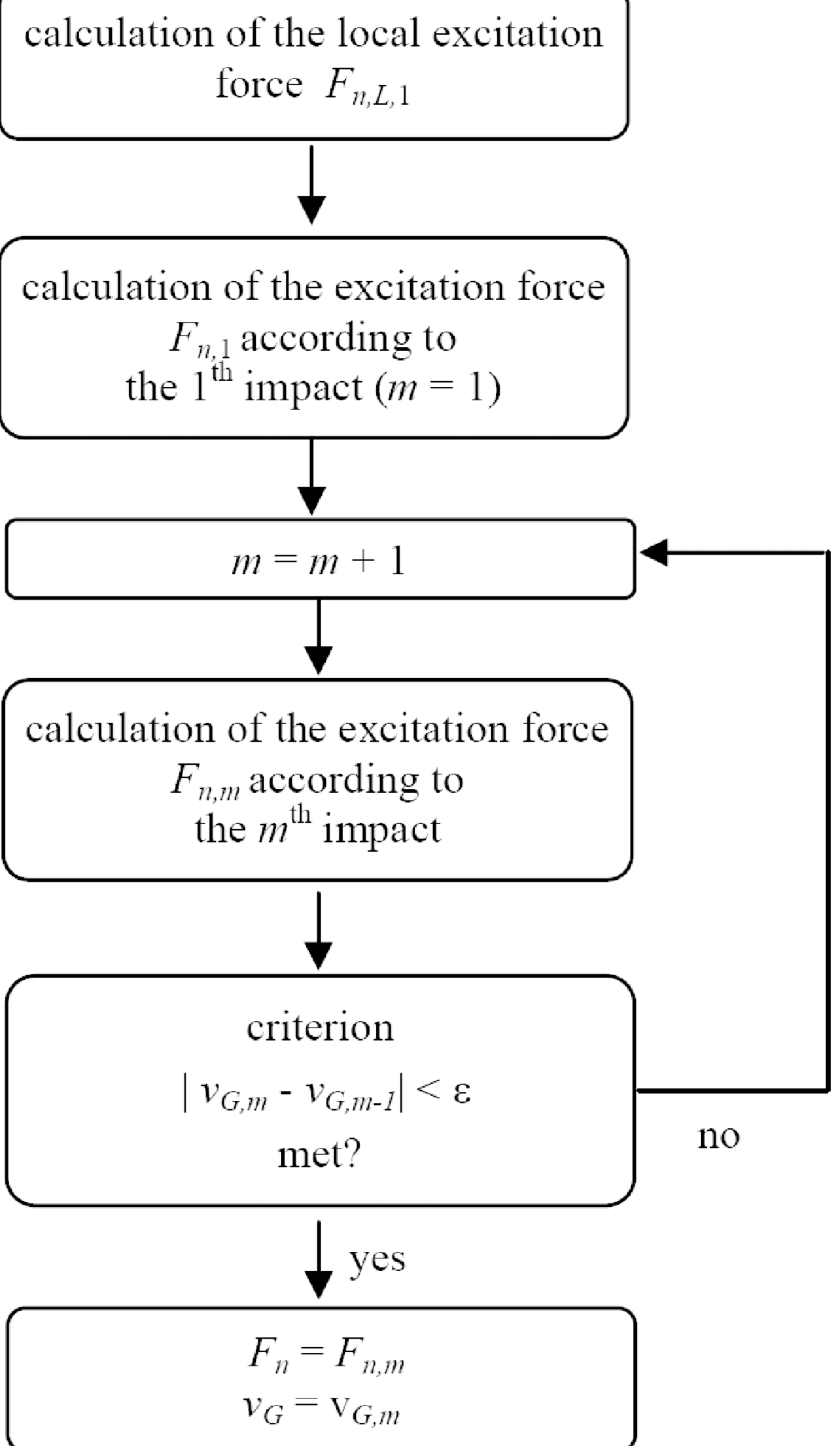

Figure 10. Iteration scheme for calculating the spectrum of the excitation 


\section{RESULTS}

\subsection{Examples for the computation in time and frequency domain}

As a first example the excitation force in time and frequency domain is computed for a tapping machine standing on a single degree of freedom system with an additional flexible covering on top. The system and the associated input data for the calculation are shown in Table 4.

Table 4. Model and input data for the calculation

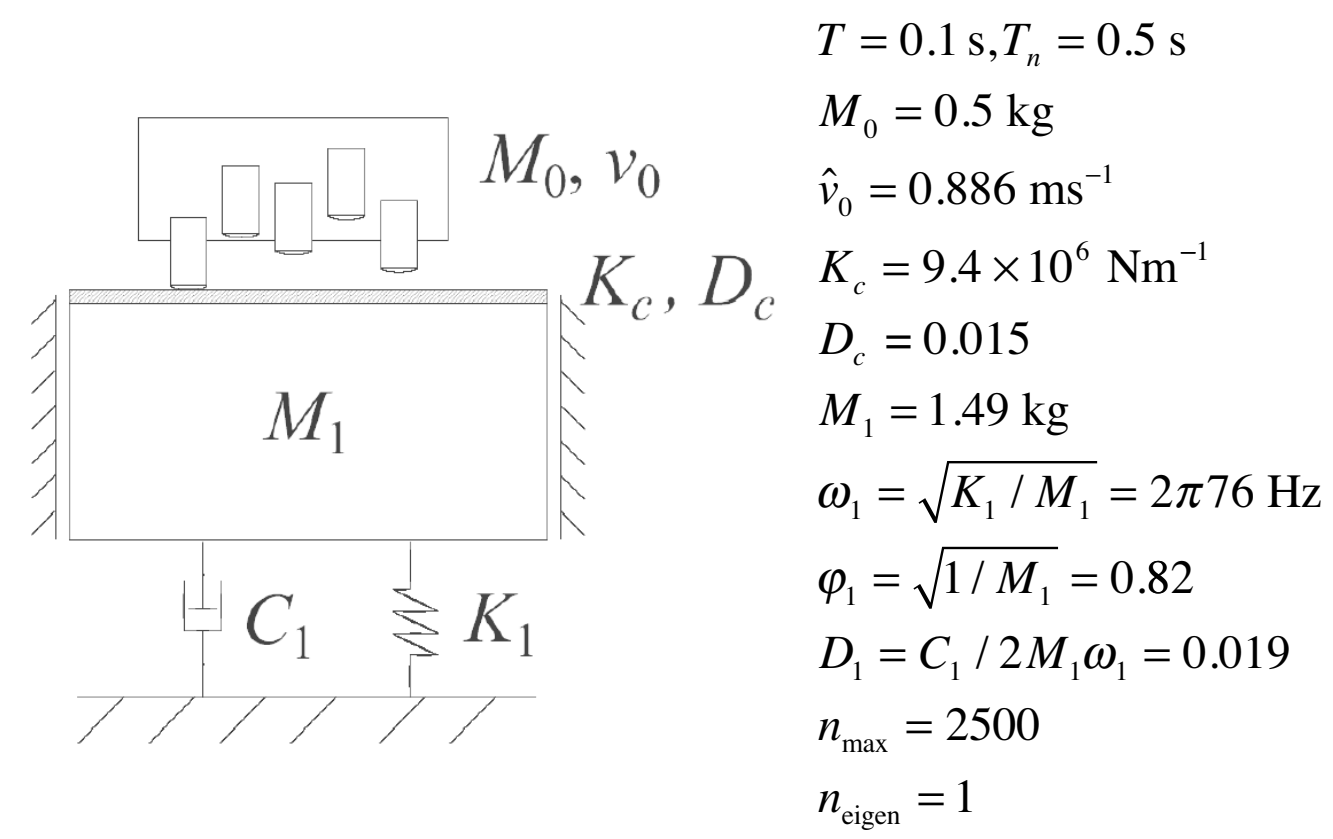

Equation (20) was used for the calculation for the first 10 hammer impacts in the time domain. Figure 11, curve (i) shows the calculation results for the first and second impact as well as the change of the maximum of the excitation force $\Delta F_{c, \text { max }}$ during the transient process (impact $\mathrm{m}$ from 1 to 10 ).

The calculation in the frequency domain was carried out using eqns (25) to (33). The calculation of the contact period $T_{c}$ and circular eigenfrequency $\omega_{c}$ was carried out by an approximation using equation (5).

In order to be able to compare the results, an inverse transformation into the time domain was carried out. The inverse transformed excitation force (curve (ii)) shows clear differences compared to the direct calculation in the time domain (curve (i)). The reason for this deviation is the assumption that the global admittance does not affect the contact period significantly, which was made for the sake of simplification (see equations (24) and (25). If however the calculation is carried out with the correct contact period (curve (iii)), a good correlation results.

Comparing the results in the frequency domain (curves (ii) and (iii) in Figure 11 below) one recognizes that the deviations resulting from the simplification are only noticeable in the high frequency range which is not relevant for this project. Thus the simplified calculation method is a good approximation for the lower frequency range.

The presentation of the transient process (Figure 11(c)) illustrates the effect of the relative velocity between component and hammer. In this example the difference between the maximum excitation force $\left(\Delta F_{c, \max }\right)$ at the 1 st impact $\left(M_{1}\right.$ still at rest) and 
the $10^{\text {th }}$ impact $\left(M_{1}\right.$ in steady state) is approx. $250 \mathrm{~N}$, which is approx. $15 \%$ of the excitation force.

(a) first impact
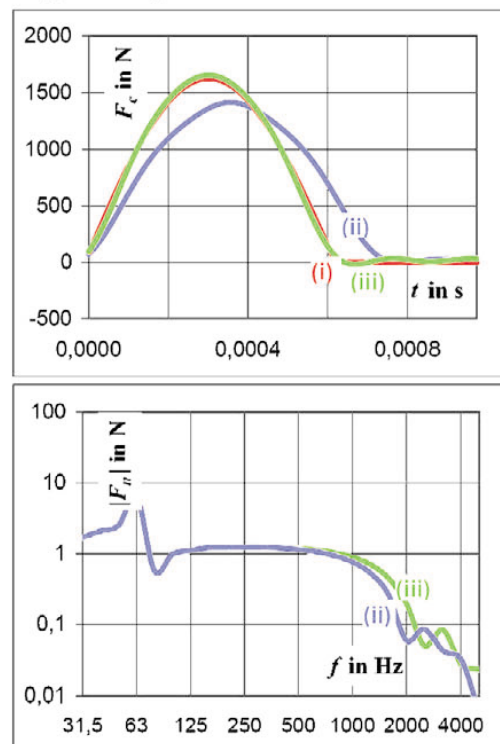

(b) second impact
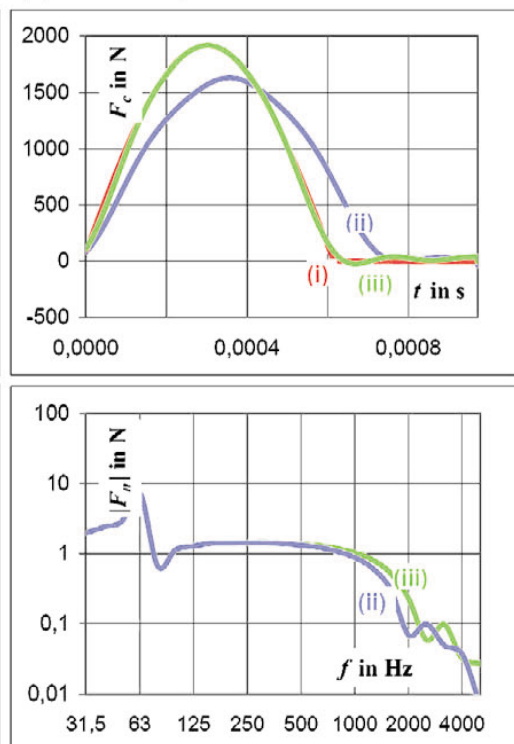

(c) transient process
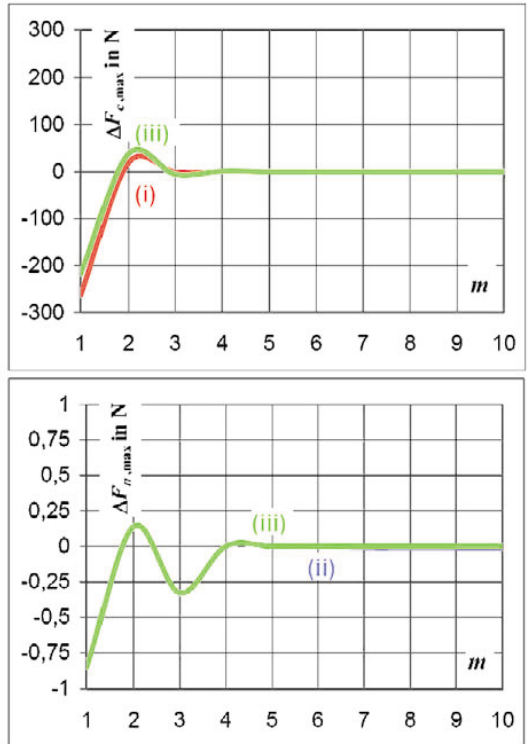

Figure 11. Transient process of excitation. Comparison of the calculations in the time domain (top) and in the frequency domain (below) for the excitation force of a hammer. (a) and (b): calculation for the $1^{\text {st }}$ and $2^{\text {nd }}$ impact. (c) the change of the maximum excitation force $\Delta F_{\max }$ during the transient process. The calculation was carried out: (i) in the time domain using equation (20), (ii) in the frequency domain using equations (25) to (33), with subsequent inverse transformation, (iii) as in (ii) but with corrected contact period. For better comparability of the results in time and frequency domain the calculation starts, in contrast to 9 , at $t=0$ for both models.

\subsection{Comparison with measurement results}

Below, the excitation spectrum obtained by the computation is compared with measured results. The measurements were carried out in the ift Centre for Acoustics using a real time analyser (type: Norsonic 830) and a force transducer (type: B\&K 8230-003) which was placed beneath one hammer of the tapping machine. The drop height was set to $0.04 \mathrm{~m}$. The other four hammers were not in contact with the test specimen. The excitation frequency for the measurement therefore was: $2 \mathrm{~Hz}$ or $T_{n}=T=0,5 \mathrm{~s}$.

Figure 12 (a) shows the comparison of the calculated excitation spectrum with measured values for a nearly ideal elastic impact. To achieve this, a force transducer was installed on a sufficiently heavy and rigid concrete block and excited directly. Figure 12 (b) shows the comparison for a situation with a local admittance larger than zero. To achieve this, various chipboard samples (thickness 19 - $25 \mathrm{~mm}$ ) were mounted on the force transducer. The excitation was applied to the chipboard samples. Equation (25) was used for the calculation, with the following input values: 

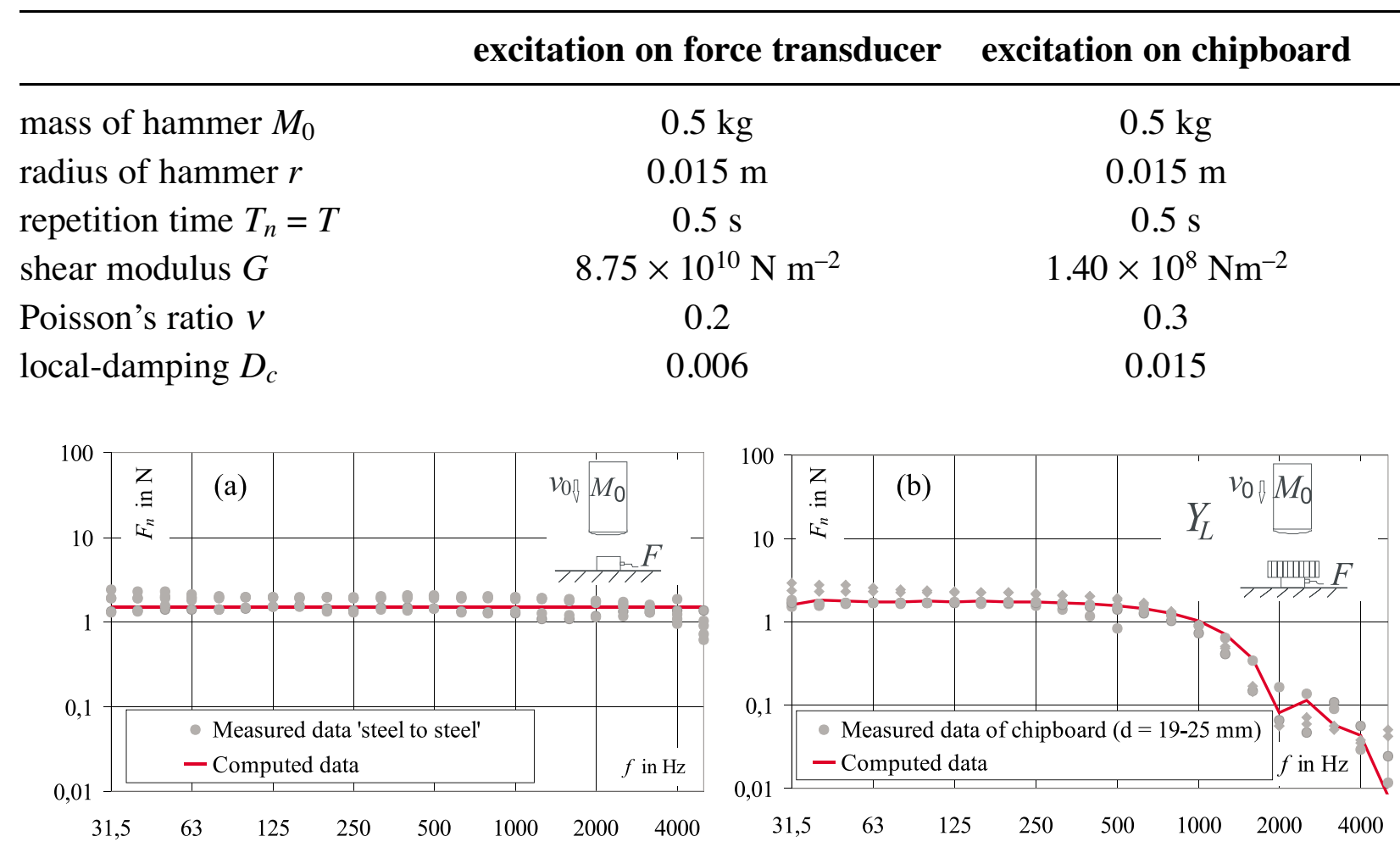

Figure 12. Comparison of computed and measured values of the spectrum of excitation force: (a) A nearly ideal elastic impact, (b) impact on chipboard.

Figure 13 shows the comparison in a configuration with a global admittance larger than zero. In this configuration, the tapping machine was standing on to a timber beam floor $\left(v_{0, t_{0}} \neq 0\right)$. The force transducer was mounted on the floor with a sufficiently large contact area and excited directly $\left(Y_{L} \rightarrow 0\right)$. The measured values for different positions of the standard tapping machine are shown on the left-hand side illustrate the influence of the different exciting points. On the right-hand side the comparison between measured and calculated values for the first eigenmode of the boarding on the timber beam floor is given.

Equations (25) to (33) were used for the calculation, with the following input parameters:

Table 6. Input data for the calculation, see Figure 13

$\begin{array}{lr}\text { mass of hammer } M_{0} & 0.5 \mathrm{~kg} \\ \text { radius of hammer } r & 0.015 \mathrm{~m} \\ \text { repetition time } T_{n}=T & 0.5 \mathrm{~s} \\ \text { shear modulus } G & 8.75 \times 10^{10} \mathrm{Nm}^{-2} \\ \text { Poisson's ratio } \mu & 0.2 \\ \text { local damping } D_{c} & 0.006 \\ \text { modal damping } D_{i} & 0.04 \\ \text { eigenfrequency } f_{0} & 80 \mathrm{~Hz} \\ \text { elongation of } \varphi\left(x_{0}, y_{0}\right) & 0.82 \\ \text { number of impacts } m & 10\end{array}$



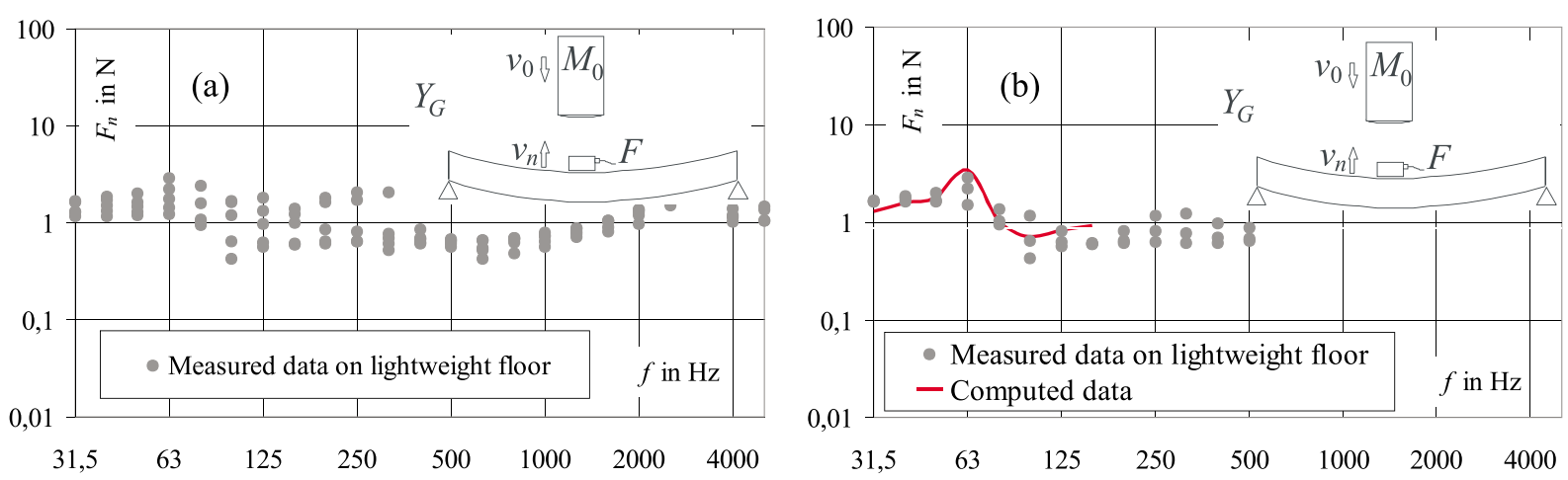

Figure 13. Comparison of the calculation with measured values of the excitation spectrum on a timber beam floor: (a) Measured values for different positions of force, (b) Comparison between measured and calculated values for the first eigenmode of the spruce boarding on a timber beam floor.

\subsection{Calculation for a timber beam floor}

In this final example, the excitation force is calculated for a timber beam floor consisting of timber beams and $22 \mathrm{~mm}$ thick chipboard. In the computation, done with the Finite Element code AdhoC [13], the structure is discretized with a fully threedimensional approach, where anisotropic high-order solid finite elements are applied allowing different polynomial degrees for each direction of the element [12]. The contact conditions between the beams and the chipboard where modelled in several ways varying stepwise between clamped and simply supported. The modelling was validated by comparison of computed and measured eigenfrequencies [11]. For the calculation of the excitation force, the spatial distribution of the tapping machine positions on the floor and the different points of excitation of the different steel cylinders of the tapping machine were taken into account. Equations (25) to (33) were used for the calculation, with the following input values:

\section{Input data for the calculation, see Figure 14}

$\begin{array}{lr}\text { mass of hammer } M_{0} & 0.5 \mathrm{~kg} \\ \text { radius of hammer } r & 0.015 \mathrm{~m} \\ \text { repetition time } T_{n} & 0.5 \mathrm{~s} \\ \text { (overall-) frequency } f_{s}=1 / T & 10 \mathrm{~Hz} \\ \text { shear modulus } G & 1.40 \times 10^{8} \mathrm{Nm}^{-2} \\ \text { Poisson's ratio } \mu & 0.3 \\ \text { local damping } D_{c} & 0.015 \\ \text { number of impacts } m & 5\end{array}$

Note that in equation (31) the velocity for the point of contact of the next impact has to be calculated. The calculation was carried out for 250 eigenvalues (eigenmodes and eigenfrequencies) of the floor and for four tapping machine positions with five excitation points each. The modal damping was determined according to [11]. Figure 14 shows the four tapping machine positions on the timber beam floor and the 
calculated excitation spectra (minimum and maximum) are presented. There ocure peaks in the frequency band from $80-200 \mathrm{~Hz}$ which are higher than the low frequency limit. The peaks results out of the consideration of the relative velocity $v_{R}$ in the improved model. This phenomenon can also be observed in the measurements as shown in Figure 13.
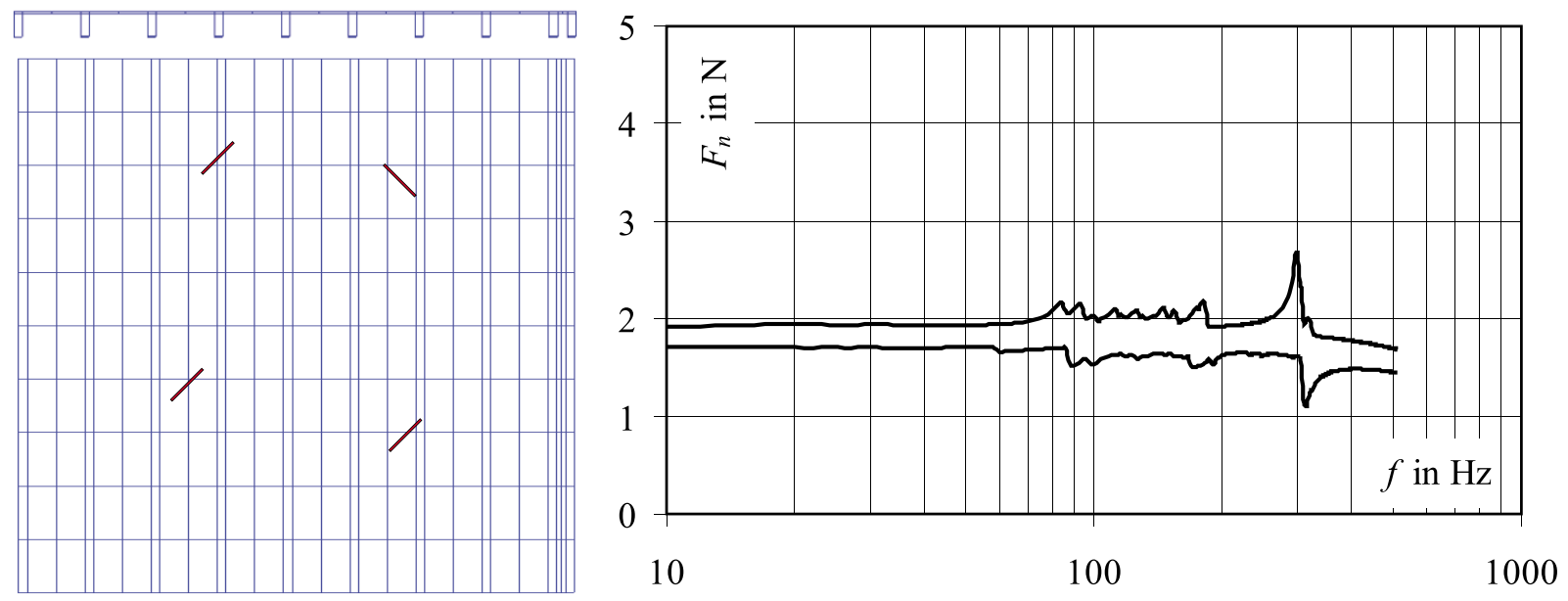

Figure 14. Calculation of the excitation spectrum on a timber beam floor. The different tapping machine positions on the floor are shown on the left; on the right the calculated spectra of the individual hammers (minimum and maximum).

Considering the global admittance and relative velocity changes the excitation force by max. $\pm 50 \%$ in this example (depending on the point of excitation/measuring and the eigenmode respectively). Effects of this order of size can only be observed for very light-weight floors, such as the timber beam floor shown here. In floor constructions with higher mass used in practice including a floating floor screed the illustrated effect plays only a minor role due to the much lower admittance of these floors.

\section{DISCUSSION}

As mentioned above, the process of optimization of lightweight floor structures has mainly been based mainly on measurements using the standard tapping machine. In order to avoid tedious measurement setups, several approaches simulating the excitation of the tapping machine have been developed.

However in all these models the relative velocity between the exciting hammer and the vibrating structure is neglected, which leads to significant errors in the case of lightweight floor structures. In this paper a more realistic model for the calculation of the excitation force in the time domain and the frequency domain was introduced. The findings in the time domain were transformed into a simplified model in the frequency domain, leading to a significant reduction of the numerical calculation effort. Special focus was placed on an accurate description of the time dependency of the force and the interaction between the impacting hammer and the vibrating surface of the floor. Still the model requires a few approximations in order to simplify the simulation. Firstly, it is assumed that the contact time is determined by the local admittance of the contact 
point only. This results in an error particularly in the higher frequency range. To investigate the effect of this approximation the simplified approach was compared to exact calculations carried out in the time domain. It was shown that the excitation spectra derived from simplified computation correlate closely with the reference values in the frequency range, relevant for this problem. Secondly, the contact point velocity of the previous impact was used in order to approximate the initial speed of the falling hammer when released from the mechanism. Here, a further refinement might be achieved by using the resulting velocity of the hammer derived from the velocity of the tapping machine at its supporting points. Finally the simulation model was validated by comparing results with measurements. A good agreement was observed and the model, whose prediction even follows resonant peaks, seems to be promising.

\section{CONCLUSION}

The simplified simulation method introduced in this paper provides a realistic model for the time dependent interaction between the tapping machine and lightweight floor constructions. The excitation spectra can be predicted within sufficient accuracy for the frequency range of interest. As the method provides a practical solution it can be applied for impact sound prediction of lightweight floors in FEM models or analytical models.

\section{ACKNOWLEDGMENTS}

The authors acknowledge the helpful discussions, in particular with Ulrich Schanda (University of applied science, Rosenheim), Andreas Mayr (University of applied science, Rosenheim) and Joachim Hessinger (ift Rosenheim). The financial support provided by the AiF and the coordination of the project by the Deutsche Gesellschaft für Holzforschung (DGfH) is also thankfully acknowledged.

\section{REFERENCES}

[1] EN ISO 140-06, Acoustics - Measurement of sound insulation in buildings and of building elements - Part 6: Laboratory measurements of impact sound insulation of floors, 1998-12.

[2] Rabold, A., Rank, E., Untersuchung der akustischen Wechselwirkungen von Holzdecken und Deckenauflage zur Entwicklung neuartiger Schallschutzmaflnahmen (Investigation of the acoustic interaction between floor and topping for developing new improvements of sound insulation); Teilbericht: Anwendung der Finiten Elemente Methode auf die Trittschallberechnung (partial report: FEM based prediction model for impact sound level), Technical report, chair for Computation in Engineering, TU München, 2009.

[3] Cremer, L., Heckl, M., Körperschall: Physikalische Grundlagen und technische Anwendungen (Structure-borne Sound: Structural vibrations and sound radiation at audio frequencies), Springer, Berlin, Heidelberg, New York, 1996.

[4] Vér, I.L., Impact noise isolation of composite floors, The journal of the Acoustic Society of America, 1971, 50, 1043-1050. 
[5] Scholl, W., Maysenhölder, W., Impact sound insulation of timber floors: Interaction between source, floor coverings and bearing floor, Journal of Building Acoustics, 1999, 6, 43-61.

[6] Petersson, B.A.T., Heckl, M., Concentrated excitation of structures, Journal of Sound and Vibration, 1996, 196, 295-321.

[7] Brunskog, J. and Hammer, P. Rigid indenter excitation of plates, Acta Acustica Acustica, 2003, 89, 460 - 470.

[8] Brunskog, J., Hammer, P., The interaction between the ISO tapping machine and lightweight floors, Acta Acustica - Acustica, 89, 2003, 296 - 308.

[9] White, R.G., Walker, J.G., Noise and Vibration, John Wiley and Sons, 1982.

[10] Rabold, A., Düster, A., Rank, E., Anwendung der Finiten Elemente Methode auf die Trittschallberechnung von Holzdecken (FEM based prediction model for the impact sound transmission of timber floors), DAGA'07, 2007, 261-262.

[11] Rabold, A., Düster, A., Berücksichtigung der Dämpfung bei der Trittschallberechnung auf Basis der Finite Elemente Methode (Consideration of damping at the FEM based impact sound prediction), DAGA'08, 2008, 411-412.

[12] Düster,A., Bröker, H., Rank, E., The p-version of the finite element method for three-dimensional curved thin walled structures, International Journal for Numerical Methods in Engineering, 52, 2001, 673-703.

[13] Düster, A.; Bröker, H.; Heidkamp, H.; Heisserer, U.; Kollmannsberger, S.; Krause, R.; Muthler, A.; Niggl, A.; Nübel, V.; Rabold, A; Rücker, M.; Scholz, D. AdhoC4 User's Guide, 2006 